\title{
Inhaltsverzeichnis
}

\author{
Vorwort zur 8. Auflage - V \\ Vorwort zur 1. Auflage — VII \\ Autoren- und Inhaltsübersicht — IX
}

Abkürzungsverzeichnis - $\mathbf{X X I}$

\section{Erster Abschnitt}

Begriff, Geschichte und Rechtsquellen des Völkerrechts

Vorbemerkung - $\mathbf{5}$

I. Begriff und Geltung des Völkerrechts - 5

1. Begriff und Besonderheiten des Völkerrechts - $\mathbf{5}$
a) Ansatzpunkte und Probleme der Begriffsbestimmung - $\mathbf{5}$
b) Begriffsgeschichte und Völkerrechtsentwicklung - 8
c) Expansion des Völkerrechts — 11
d) Kombinationsdefinition des Völkerrechts - 15
e) Abgrenzung zu anderen Rechtsgebieten - 17
f) Völkerrecht und Europarecht - 20
g) Besonderheiten des Völkerrechts - 22

2. Geltung, Grundregeln und Einheit des Völkerrechts - 26

a) Die Frage der Geltung - 26

b) Die Grundregeln des Völkerrechts - $\mathbf{3 0}$

c) Die Einheit der Völkerrechtsordnung - 34

II. Völkerrechtsgeschichte und Völkerrechtswissenschaft - $\mathbf{3 8}$

1. Geschichte und Geschichtlichkeit des Völkerrechts - 38

2. Völkerrechtswissenschaft $-\mathbf{4 8}$

III. Die Rechtsquellen des Völkerrechts - $\mathbf{5 0}$

1. In Art $\mathbf{3 8}$ IGH-Statut genannte Rechtsquellen und Erkenntnismittel - $\mathbf{5 0}$

a) Internationale Übereinkünfte (das Recht der Verträge) - $\mathbf{5 0}$

b) Völkergewohnheitsrecht - 60

c) Allgemeine Rechtsgrundsätze - 64

d) Richterliche Entscheidungen, Lehrmeinung - 66

2. Entwicklungen außerhalb von Art 38 IGH-Statut -67

3. Rangverhältnis und Kodifikation der Rechtsquellen $-\mathbf{7 0}$

\section{Zweiter Abschnitt}

Völkerrecht und staatliches Recht

I. Einführung in die Grundlagen -79

II. Verfassungsrechtliche Grundentscheidungen für die Stellung Deutschlands in der internationalen Ordnung - $\mathbf{8 2}$ 
1. Das Bekenntnis zu Frieden und Menschenrechten - $\mathbf{8 3}$

2. Die „Offenheit“ und die „Völkerrechtsfreundlichkeit“ $-\mathbf{8 6}$

3. Die Integrationsorientierung - $\mathbf{8 8}$

III. Das Verhältnis des Völkerrechts zur staatlichen Rechtsordnung:

Grundbegriffe und Grundpositionen — 92

1. Der Theorienstreit $-\mathbf{9 2}$

2. Die Mechanismen - 95

3. Geltung und Anwendbarkeit - 96

4. Völkerrechtliche Rechtsfolgen mangelnder Umsetzung — 97

IV. Völkerrecht und deutsches Recht — 98

1. Zur Einführung: Das Verhältnis von Völkerrecht und staatlichem Recht nach den Rechtsordnungen einzelner Staaten — 98

2. Die völkerrechtlichen Verträge - $\mathbf{1 0 7}$

a) Überblick zu den grundgesetzlichen Vorgaben - 107

b) Bund und Länder als Parteien völkerrechtlicher Verträge und als Mitwirkende beim Vertragsschluss - 108

c) Repräsentation nach außen: Die Befugnisse des Bundespräsidenten - $\mathbf{1 1 2}$

d) Die Rolle der Bundesregierung — $\mathbf{1 1 4}$

e) Das Erfordernis der Mitwirkung von Bundestag und Bundesrat - 117

f) Die Form des Bundesgesetzes - $\mathbf{1 2 5}$

g) Der Rang und die Wirkung völkerrechtlicher Verträge im deutschen Recht - 128

h) Die europäischen Gründungsverträge im deutschen Recht — 130

i) Die Übertragung von Hoheitsrechten im Übrigen — 135

3. Das Völkergewohnheitsrecht - $\mathbf{1 3 6}$

a) Völkergewohnheitsrecht und „allgemeine Regeln des Völkerrechts“— 137

b) Der Rang und die Wirkung gewohnheitsrechtlich geltender allgemeiner Regeln des Völkerrechts im deutschen Recht — 141

c) Die Normverifikation durch das Bundesverfassungsgericht - 146

4. Allgemeine Rechtsgrundsätze - $\mathbf{1 4 7}$

5. Recht Internationaler Organisationen - 148

V. Bilanz: Völkerrecht als Maßstab deutscher Staatsgewalt - 151

1. Gesetzgebung — $\mathbf{1 5 1}$

2. Regierung und Verwaltung $-\mathbf{1 5 2}$

3. Gerichte - $\mathbf{1 5 5}$

\section{Dritter Abschnitt}

\section{Der Staat und der Einzelne als Völkerrechtssubjekte}

I. Die Rechtsträger im Völkerrecht, ihre Organe und die Regeln des zwischenstaatlichen Verkehrs - $\mathbf{1 7 0}$

1. Rechtsträger und Handelnde im Völkerrecht - $\mathbf{1 7 0}$
a) Entwicklung - $\mathbf{1 7 0}$
b) Einteilung der Völkerrechtssubjekte - $\mathbf{1 7 1}$ 

c) Internationale Organisationen - $\mathbf{1 7 2}$
d) Der Einzelne - $\mathbf{1 7 3}$
e) Völker — 179
f) Minderheiten $-\mathbf{1 7 9}$
g) Sonstige - $\mathbf{1 8 0}$

2. Organe der Völkerrechtssubjekte und Regeln des zwischenstaatlichen Verkehrs - 183
a) Zentrale Organe - $\mathbf{1 8 3}$
b) Diplomatische Missionen - $\mathbf{1 8 8}$
c) Konsulate - 194
d) Sonderbotschafter $\mathbf{1 9 6}$
e) Vertretung bei Internationalen Organisationen — 197
f) Diplomatisches Asyl — 197

II. Der Staat als primäres Völkerrechtssubjekt — 199

1. Der Staat - $\mathbf{1 9 9}$
a) Die Elemente des Staates - 199
b) Die staatliche Souveränität $-\mathbf{2 0 2}$
c) Die Gleichheit der Staaten — 204

2. Das Staatsvolk - $\mathbf{2 1 8}$
a) Die Staatsangehörigkeit $-\mathbf{2 1 8}$
b) Diplomatischer Schutz - 229
c) Das Selbstbestimmungsrecht der Völker - 235

3. Das Staatsgebiet - 239
a) Territoriale Souveränität und Gebietshoheit - 239
b) Erwerb und Verlust von Staatsgebiet - $\mathbf{2 4 0}$
c) Umfang des Staatsgebiets $-\mathbf{2 4 3}$

4. Die Staatsgewalt $-\mathbf{2 4 5}$
a) Umfang der Staatsgewalt und Neutralität - $\mathbf{2 4 5}$
b) Staatsähnliche Völkerrechtssubjekte - $\mathbf{2 4 7}$

5. Entstehung und Untergang von Staaten - $\mathbf{2 5 0}$
a) Grundlagen - $\mathbf{2 5 0}$
b) Die völkerrechtliche Anerkennung - 251
c) Die neuen Staaten in Mittel- und Osteuropa - 253

6. Die Staatensukzession - $\mathbf{2 5 5}$
a) Begriff und Rechtsgrundlage - $\mathbf{2 5 5}$
b) Die Nachfolge in völkerrechtliche Verträge - 256
c) Die Nachfolge in Staatsvermögen, Staatsarchive, Staatsschulden und Haftungsansprüche -257
d) Staatennachfolge und Staatsangehörigkeit - 259

7. Die Rechtslage Deutschlands in Geschichte und Gegenwart — 260
a) Die Teilung Deutschlands -261
b) Die Vereinigung Deutschlands - $\mathbf{2 6 2}$
c) Die Regelungen über die Staatennachfolge - 264

III. Der Einzelne im Völkerrecht — $\mathbf{2 6 5}$

1. Der Menschenrechtsschutz auf universeller Ebene - $\mathbf{2 6 5}$
a) Einführung - $\mathbf{2 6 5}$
b) Die Allgemeine Erklärung der Menschenrechte -269 
c) Die Menschenrechtspakte v 19.12.1966 - 271

d) Spezielle Konventionen zum Schutz der Menschenrechte - 272

2. Der Menschenrechtsschutz auf regionaler Ebene - $\mathbf{2 7 4}$
a) Der Europarat - $\mathbf{2 7 4}$
b) Die Europäische Konvention zum Schutze der Menschenrechte und Grundfreiheiten — 275
c) Die Europäische Sozialcharta - 284
d) Die Menschenrechte im Rahmen der KSZE/OSZE — 285
e) Der Menschenrechtsschutz in Amerika - $\mathbf{2 8 6}$
f) Die Menschenrechte in Afrika — $\mathbf{2 8 8}$
g) Die Menschenrechte in der Arabischen Liga - $\mathbf{2 8 9}$

3. Das völkerrechtliche Fremdenrecht $-\mathbf{2 8 9}$
a) Einführung - $\mathbf{2 8 9}$
b) Die Einreise von Ausländern — 291
c) Die Rechtsstellung von Ausländern - 292
d) Die Ausweisung von Ausländern — 293

4. Das Recht auf Asyl — 297
a) Der Begriff des Flüchtlings - 297
b) Das Recht auf Asyl - 299
c) Der Grundsatz des Non-Refoulement - $\mathbf{3 0 0}$
d) Maßnahmen im Rahmen des Europarats - $\mathbf{3 0 3}$
e) Flüchtlinge in den EU-Mitgliedstaaten - 304

5. Die Auslieferung - 309
a) Einführung - 309
b) Grundzüge der Auslieferungsverträge - $\mathbf{3 1 0}$
c) Die political offence exception - $\mathbf{3 1 1}$
d) Auslieferung und EMRK - $\mathbf{3 1 1}$
e) Gewaltsames Verbringen in den Gerichtsstaat — 311

6. Der völkerrechtliche Minderheitenschutz - $\mathbf{3 1 2}$
a) Einführung - $\mathbf{3 1 2}$
b) Der Minderheitenschutz auf universeller Ebene - $\mathbf{3 1 4}$
c) Regionaler Minderheitenschutz - 315

\section{Vierter Abschnitt}

\section{Die Internationalen und die Supranationalen Organisationen}

I. Geschichte und Bedeutung der Internationalen Organisationen - 328

1. Die Internationalen Organisationen als unverzichtbare Elemente der internationalen Zusammenarbeit - $\mathbf{3 2 8}$

2. Die Wurzeln: Von den Friedenskongressen und Verwaltungsunionen zum Völkerbund - 328

3. Die Entwicklung nach $1945-331$

4. Definitionen und Abgrenzungen - 333

5. Der Einfluss der Internationalen Organisationen auf das Völkerrecht - 335

II. Das Recht der Internationalen Organisationen - 341 
1. Entstehung und Untergang Internationaler Organisationen - $\mathbf{3 4 1}$
a) Der völkerrechtliche Gründungsakt - $\mathbf{3 4 1}$
b) Der Gründungsvertrag als „Verfassung“ der Internationalen Organisationen - 342
c) Auslegung und Änderung des Gründungsvertrags - 343
d) Untergang von Internationalen Organisationen - 348
e) Sukzessionsfragen - $\mathbf{3 5 0}$

2. Die Mitgliedschaft in Internationalen Organisationen - $\mathbf{3 5 2}$
a) Erwerb der Mitgliedschaft - $\mathbf{3 5 2}$
b) Mitgliedschaftsrechte und -pflichten - 357
c) Beendigung der Mitgliedschaft - $\mathbf{3 5 8}$
d) Beeinträchtigungen der Mitgliedschaftsrechte - 362
e) Abgestufte Formen der Mitgliedschaft - 364
f) Nichtmitglieder $-\mathbf{3 6 6}$

3. Die Rechtsstellung Internationaler Organisationen - $\mathbf{3 6 8}$
a) Völkerrechtsfähigkeit — $\mathbf{3 6 8}$
b) Völkerrechtliche Handlungsfähigkeit — 369
c) Immunitäten, Privilegien, Verhältnis zum Sitzstaat - 374
d) Innerstaatliche Rechts- und Geschäftsfähigkeit - $\mathbf{3 7 8}$
e) Qualifikation des internen Organisationsrechts - 379

4. Die Organe und ihre Willensbildung - $\mathbf{3 8 1}$
a) Allgemeine Strukturen - $\mathbf{3 8 1}$
b) Die Hauptorgane von UN, Europarat und EU im Vergleich - 384
c) Die Hauptorgane der UN - $\mathbf{3 8 5}$
d) Wandlungen der Organstrukturen und Zwischen-Organ- Verhältnis — 416

5. Aufgaben und Befugnisse Internationaler Organisationen - 419
a) Allgemeines $-\mathbf{4 1 9}$
b) Festlegung der Kompetenzausstattung, Prinzip der begrenzten Ermächtigung, ultra vires-Handeln -420
c) Respektierung der inneren Zuständigkeit der Mitglied- staaten -423
d) Bindung an menschenrechtliche Mindeststandards - 424
e) Handlungsinstrumentarium $-\mathbf{4 2 6}$
f) Die wichtigsten Aufgabenfelder der UN $-\mathbf{4 2 8}$

6. Die Finanzierung Internationaler Organisationen - 436
a) Einnahmen - 437
b) Ausgaben und Budgetierung — 441
c) Rechnungskontrolle und Sanktionen - 442

III. Die UN-Familie - $\mathbf{4 4 3}$
1. Allgemeines $-\mathbf{4 4 3}$
2. UN mit Haupt- und Nebenorganen - $\mathbf{4 4 4}$
3. Sonderorganisationen -444

IV. Europarat - $\mathbf{4 4 8}$

1. Allgemeines und Entstehung - 448

2. Organe $-\mathbf{4 5 0}$

3. Aufgaben, Grundsätze, Aktivitäten 451 
V. Europäische Union - $\mathbf{4 5 5}$

1. Supranationale Organisation $-\mathbf{4 5 5}$

2. Völkerrechtssubjektivität und völkerrechtliche Handlungsfähigkeit - 456

3. Unionszuständigkeiten und Grundrechtsschutz — 457

4. Entwicklungsperspektiven $-\mathbf{4 5 9}$

\section{Fünfter Abschnitt}

\section{Raum und Umwelt im Völkerrecht}

\section{Vorbemerkung - $\mathbf{4 7 4}$}

I. Der Raum im Völkerrecht $-\mathbf{4 7 4}$

1. Die Raumordnung des Völkerrechts im Überblick - $\mathbf{4 7 4}$
a) Territoriale Souveränität und Gebietshoheit - 474
b) Staatsgebiet und Nichtstaatsgebiet als Grundkategorien - $\mathbf{4 7 6}$
c) Nichtstaatsgebiet: Funktionshoheits- und Staatengemeinschafts- räume - 477

2. Staatsgebiet: Raum territorial radizierter, umfassender Hoheitsgewalt - 481
a) Wesen und Grenzen: Gebietshoheit im Rahmen des Völker- rechts -481
b) Erwerb und Verlust von Staatsgebiet $-\mathbf{4 8 6}$
c) Luftraum: Lufthoheit und Luftfreiheiten - $\mathbf{4 8 8}$
d) Maritimes Aquitorium: Innere Gewässer, Archipelgewässer, Küsten- meer -493

3. Nichtstaatsgebiet (1): Küstenstaatliche Funktionshoheitsräume - $\mathbf{5 0 3}$
a) Wesen und Grenzen: Räume begrenzter Hoheitsbefugnisse
503
b) Anschlusszone: Raum polizeilicher Kontrollrechte - $\mathbf{5 0 4}$
c) Ausschließliche Wirtschaftszone: Ressourcenorientierter Raum sui generis - $\mathbf{5 0 5}$
d) Festlandsockel: Erforschungs- und Ausbeutungsmonopol aus Küstenlage - $\mathbf{5 0 9}$

4. Nichtstaatsgebiet (2): Globale Staatengemeinschaftsräume - $\mathbf{5 1 4}$
a) Wesen und Grenzen: Gemeinschaftsbezogene Forschungs- und Nutzungsfreiheit $-\mathbf{5 1 4}$
b) Hohe See: Raum rechtlich geordneter Freiheit - $\mathbf{5 1 5}$
c) Tiefseeboden (,Gebiet“): Internationalisiertes Menschheits- erbe - $\mathbf{5 1 9}$
d) Weltraum: Kooperationsverpflichtete Freiheit und Gleichheit - $\mathbf{5 2 3}$
e) Antarktis: Eher „Weltpark“ als „Klubraum - $\mathbf{5 2 8}$

II. Die Umwelt im Völkerrecht $-\mathbf{5 3 2}$

1. Das Völkerrecht des Umweltschutzes im Überblick — $\mathbf{5 3 2}$

2. Die Entwicklung des Umweltvölkerrechts - $\mathbf{5 3 4}$
a) Die Ausgangspunkte: Nachbarrecht und Artenschutz- abkommen $-\mathbf{5 3 5}$
b) Die großen Umweltkonferenzen - $\mathbf{5 3 7}$

3. Allgemeine Prinzipien des Umweltvölkerrechts - $\mathbf{5 4 3}$ 

a) Das Trail Smelter-Prinzip und der Präventionsgrundsatz - $\mathbf{5 4 5}$
b) Das Gebot der ausgewogenen Mitnutzung grenzübergreifender Ressourcen - $\mathbf{5 4 8}$
c) Das Vorsorge- und das Verursacherprinzip - $\mathbf{5 4 9}$
d) Das Leitbild der nachhaltigen Entwicklung - 552

4. Umweltschutz und Individualrechte $-\mathbf{5 5 4}$

5. Bereichsspezifische Instrumente des Umweltvölkerrechts - $\mathbf{5 5 7}$
a) Schutz der Binnengewässer - $\mathbf{5 5 8}$
b) Schutz des Meeres - $\mathbf{5 6 4}$
c) Schutz der Luft und Bekämpfung des Klimawandels - $\mathbf{5 7 0}$
d) Arten- und Biodiversitätsschutz $-\mathbf{5 7 9}$
e) Schutz vor Abfällen und Schadstoffen - $\mathbf{5 8 3}$

\section{Sechster Abschnitt \\ Wirtschaft und Kultur}

I. Die Wirtschaft im Völkerrecht — $\mathbf{5 9 3}$

1. Das Völkerrecht der wirtschaftlichen Zusammenarbeit im Überblick — $\mathbf{5 9 3}$
a) Internationales Wirtschaftsrecht - $\mathbf{5 9 7}$
b) Weltwirtschaftsordnung - $\mathbf{5 9 8}$

2. Standards des internationalen Wirtschaftsrechts $-\mathbf{6 0 0}$
a) Grundsatz der Nichtdiskriminierung - 600
b) Meistbegünstigungsgrundsatz -602
c) Grundsatz der Inländergleichbehandlung - 605
d) Reziprozität, Fairness - 606
e) Recht auf Entwicklung, Nachhaltigkeit - 609
f) Rationalität, Transparenz, Good Governance - 614

3. Schutz fremden Eigentums und Status transnationationaler Unternehmen - 618
a) Schutz von Auslandsinvestitionen - 618
b) Transnationale Unternehmen - $\mathbf{6 2 4}$

4. Welthandelsordnung -630
a) Instrumente des freien Welthandels: GATT und WTO $-\mathbf{6 3 0}$
b) Handelsbezogene Investitionsmaßnahmen - 646
c) Instrumente regionaler wirtschaftlicher Integration - 647
d) Weitere handelsbezogene Instrumente - 651

5. Internationales Steuerrecht - $\mathbf{6 5 2}$

6. Das Völkerrecht der wirtschaftlichen Entwicklung - 654
a) Grundlagen und Entwicklung - 654
b) Internationaler Währungsfonds und Weltbankgruppe - 655
c) Rohstoffabkommen und Cotonou-Abkommen - 659

II. Die Kultur im Völkerrecht -663

1. Kulturgüterrecht im Überblick - 663

2. Informationsfreiheit und Kulturordnung - 665

3. Kulturelle Belange als Schranke der Waren- und Dienstleistungsfreiheiten - 666 
4. Recht auf Sprache -669

5. Kulturgüter und ihr Schutz -670
a) Definition des Kulturguts - 673
b) Rechtsträger von Kulturgütern $-\mathbf{6 7 5}$
c) Kulturgüter in bewaffneten Konflikten - $\mathbf{6 7 8}$
d) Regelung für Friedenszeiten - 681
e) Rückführung von Kulturgut - 685
f) Kulturgüterschutz im Meer - 687
g) Archivgut, Archäologie, Architektur -688

\section{Siebenter Abschnitt}

Verantwortlichkeit, Völkerstrafrecht, Streitbeilegung und Sanktionen

I. Völkerrechtsverletzungen als Hauptthema des Abschnitts -698

II. Internationale Verantwortlichkeit -698

1. Begriff und Terminologie - $\mathbf{6 9 8}$

2. Grundlagen und Konzept der Staatenverantwortlichkeit -699
a) Rechtliches Fundament - 699
b) Umriss - $\mathbf{7 0 0}$
c) Ansätze zur Ausweitung des Konzepts - 702

3. Einzelfragen $-\mathbf{7 0 6}$
a) Zurechnungsprobleme der Staatenverantwortlichkeit - $\mathbf{7 0 6}$
b) Ausschluss der Staatenverantwortlichkeit - $\mathbf{7 0 8}$
c) Rechtsfolgen der Staatenverantwortlichkeit - $\mathbf{7 1 0}$

4. Die Verantwortlichkeit Internationaler Organisationen - $\mathbf{7 1 2}$
a) Praktische Bedeutung - 712
b) Einschlägige Regeln -712

III. Völkerstrafrecht -714

1. Das Konzept $-\mathbf{7 1 4}$
a) Begriff und Kriterien - $\mathbf{7 1 4}$
b) Inhaltlicher Bezug zu Frieden und Sicherheit der Menschheit — 716

2. Grundelemente der Normierung - $\mathbf{7 2 0}$
a) Abgrenzung in Bezug auf die staatliche Strafgewalt $-\mathbf{7 2 0}$
b) Ausgestaltung der Tatbestände und der Strafbarkeits- voraussetzungen $-\mathbf{7 2 1}$
c) Allgemeine Strafrechtsprinzipien und Strafsanktionen - $\mathbf{7 2 3}$
d) Die Strafgewalt internationaler Gerichte, insbesondere des IStGH -724
e) Strafverfahren -725

3. Bilanz -726

IV. Streitbeilegung -726

1. Die Pflicht zur friedlichen Streitbeilegung - $\mathbf{7 2 6}$
a) Rechtliches Fundament - $\mathbf{7 2 6}$
b) Rechtssystematischer Standort - 727
c) Gegenstand - $\mathbf{7 2 8}$
d) Inhalt und Grenzen — 729 
2. Die Mittel der Streitbeilegung im Überblick — 729

3. Diplomatische Verfahren - 731
a) Verhandlungen und Konsultationen - 731
b) Verfahren mit Drittbeteiligung - 731

4. Schiedsgerichte $-\mathbf{7 3 3}$
a) Allgemeine Kennzeichnung - 733
b) Erscheinungsformen - 734
c) Einzelfragen des schiedsgerichtlichen Verfahrens - 735
d) Praktische Bedeutung - 735

5. Der Internationale Gerichtshof $-\mathbf{7 3 6}$
a) Grundlagen - 736
b) Zuständigkeit - 737
c) lus standi $-\mathbf{7 4 0}$
d) Entscheidungsmaßstab $-\mathbf{7 4 0}$
e) Organisation - 741
f) Grundzüge des Streitverfahrens - 742
g) Praktische Bedeutung - 744

V. Sanktionen - $\mathbf{7 4 5}$

1. Allgemeine Kennzeichnung - 745

a) Begriff und Abgrenzung - 745

b) Beschränkung des Begriffs auf Maßnahmen Internationaler Organisationen -746

2. Erscheinungsformen -746

3. Einzelprobleme - $\mathbf{7 4 8}$

a) Die Repressalie - $\mathbf{7 4 8}$

b) Wirtschaftssanktionen -751

\section{Achter Abschnitt \\ Friedenssicherung und Kriegsrecht}

Vorbemerkung - 762

I. Völkerrechtliche Verhinderung von Gewalt (ius contra bellum) - 762

1. Das rechtliche Verbot von Gewalt - $\mathbf{7 6 2}$
a) Entwicklung - 762
b) Verbotene Gewalt - 766
c) Rechtfertigungsgründe für Gewalt - $\mathbf{7 7 4}$
d) Rechtliche Konsequenzen einer Verletzung und Durchsetzung des Gewaltverbots - 785
e) Funktion und Bedeutung des Gewaltverbots - 788

2. Rahmenbedingungen des Gewaltverbots - $\mathbf{7 9 0}$
a) Friedliche Streitbeilegung und friedlicher Wandel $-\mathbf{7 9 0}$
b) Das System der kollektiven Sicherheit - 791
c) Rüstungskontrolle und Abrüstung - $\mathbf{8 0 8}$

II. Völkerrechtliche Eingrenzung von Gewalt - Das Recht bewaffneter Konflikte (ius in bello) - $\mathbf{8 1 5}$

1. Grundlage und Entwicklung - $\mathbf{8 1 5}$ 
2. Die Beziehungen zwischen den Konfliktparteien - $\mathbf{8 2 0}$
a) Der Anwendungsbereich des völkerrechtlichen Kriegsrechts - der internationale bewaffnete Konflikt $-\mathbf{8 2 0}$
b) Allgemeine Grundsätze und Landkriegsrecht — 821
c) Seekrieg - $\mathbf{8 4 1}$
d) Luftkrieg und Militarisierung des Weltraums - $\mathbf{8 4 3}$
e) Wirtschaftskrieg - 844
f) Durchsetzung des ius in bello - $\mathbf{8 4 4}$

3. Konfliktparteien und dritte Staaten (Neutralitätsrecht) $-\mathbf{8 5 4}$
a) Grundlagen - $\mathbf{8 5 4}$
b) Unverletzlichkeit des neutralen Gebiets - $\mathbf{8 5 8}$
c) Neutralitätspflichten der Nichtteilnehmer - $\mathbf{8 5 8}$
d) Landkrieg -859
e) Seekrieg $-\mathbf{8 6 0}$
f) Luftkrieg - $\mathbf{8 6 2}$

4. Der nichtinternationale bewaffnete Konflikt $-\mathbf{8 6 4}$

5. Die Internationalisierung nichtinternationaler Konflikte $-\mathbf{8 7 0}$

III. Rechtliche Steuerung des Wegs vom bewaffneten Konflikt zur friedlichen Normalität (ius post bellum) $-\mathbf{8 7 2}$ 